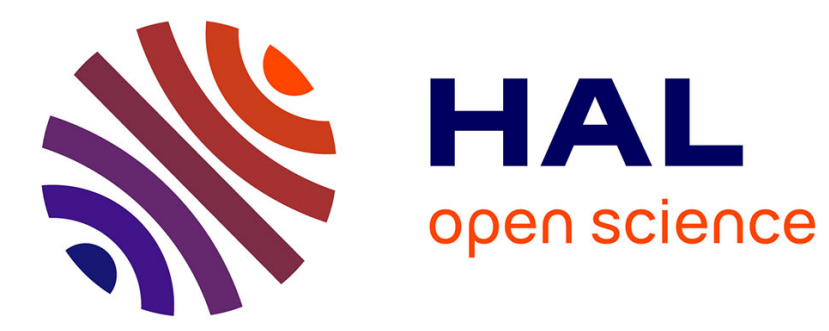

\title{
EXPERTS ou MÉDIATEURS ?: Les Professionnels de l'urbanisme
}

Gilles Verpraet

\section{To cite this version:}

Gilles Verpraet. EXPERTS ou MÉDIATEURS ?: Les Professionnels de l'urbanisme. Les Annales de la Recherche Urbaine, 1988, 37, pp 98-105. hal-00332404

\section{HAL Id: hal-00332404 \\ https://hal.science/hal-00332404}

Submitted on 22 Oct 2008

HAL is a multi-disciplinary open access archive for the deposit and dissemination of scientific research documents, whether they are published or not. The documents may come from teaching and research institutions in France or abroad, or from public or private research centers.
L'archive ouverte pluridisciplinaire HAL, est destinée au dépôt et à la diffusion de documents scientifiques de niveau recherche, publiés ou non, émanant des établissements d'enseignement et de recherche français ou étrangers, des laboratoires publics ou privés. 
EXPERTS

OU

MÉDIATEURS ?

LES PROFESSIONNELS

DE LURBANISME

Gilles Verpraet

L'urbanisme français a longtemps été caractérisé par la place importante de l'Etat central et de ses politiques publiques dans sa mise en œuvre. Les transformations des dix dernières années ont accru le rôle des élus locaux, la présence des associations de quartier dans la définition et la négociation des projets. Il en résulte des transformations importantes de l'exercice professionnel des urbanistes : la multiplicité des partenaires accroit la part de la médiation entre les acteurs de l'urbanisme.

Pour les professionnels de l'urbanisme, les questions sont nombreuses et complexes. Le développement des exigences de concertation, de négociation dans l'intervention urbanistique s'accompagne d'un resserrement des exigences financières. La diversification des rôles professionnels (étude préalable et concertation, gestion et anticipation) accroît la difficulté de fixer des statuts professionnels de référence. Quelles sont les nouvelles conditions pour l'émergence et la formalisation de projets urbanistiques, perçues et interprétées par les professionnels de l'urbanisme?

\section{LES ORIENTATIONS PROFESSIONNELLES DES URBANISTES}

Les premières codifications de l'exercice professionnel de l'urbanisme sont liées à l'intervention des services de l'Etat où prédomine la définition de l'expert autorisé, dans sa capacité à mettre en œuvre et à interpréter un règlement d'urbanisme'.

L'homme de l'art est jugé compétent pour mettre en œuvre les plans d'embellissement et d'extension, sans autre précision sur les formations préalables, architectes, ingénieurs, ou membres de la Société Française des Urbanistes naissante. Dans les années 1940/1945, la codification publique des urbanistes est resserrée sur la définition des urbanistes fonctionnaires de l'Etat et sur celle de l'Ordre des architectes, c'est-à-dire la délimitation d'experts centraux, codifiée en termes de formation et de recrutement.

Dans les années 1960/75, la gestion publique de la croissance urbaine, la mise en place des services de transport, le développement du logement social mettent l'accent sur le rôle des urbanistes dans la gestion publique, sous ses différents aspects d'allocation des ressources publiques, de financement des services urbains, de redistribution sociale ${ }^{2}$ et de soutien au développement de l'activité économique.

Les conflits écologiques et le mouvement des associations de défense de l'environnement, dans les années 1975-80, ont accru les exigences de participation publique dans les procédures de l'urbanisme. La place des habitants, des associations, tend à redéfinir la réflexion professionnelle et leurs modes d'action vers des rôles de médiation, de négociation et de mise en relation des acteurs de l'urbanisme.

Les processus de décentralisation de l'urbanisme en cours, où les maires et les communes acquièrent une responsabilité sur la définition de leur politique urbaine, renversent quelque peu ces définitions centrales de l'urbaniste. Ils reposent la question des fondations historiques et synchroniques des professions de l'urbanisme.

Le nouveau contexte pour l'action des urbanistes est caractérisé par deux relations principales:

- les relations entre l'Etat central et les collectivités locales impliquent l'urbaniste dans les œuvres de la redistribution sociale : le logement social, l'implantation d'équipements publics, la liaison formation/emploi ;

- les relations entre les municipalités et leurs habitants impliquent les urbanistes dans le développement des quartiers, dans la communication municipale, dans la promotion de la participation publique.

L'action de l'Etat se déplace du recul de l'intervention publique vers un rôle de régúlation dans ces différents rapports, notamment entre municipalités et promoteurs de logement (promotion de la qualité urbaine réhabilitation, plans locaux de l'habitat) ${ }^{3}$.

\section{UNE POSITION D'INTERMÉDIAIRE}

La notion de médiation implique une réciprocité des perspectives d'analyse entre l'Etat et la société civile, pour de nombreuses professions intermédiaires. Dans ces recherches sur les professions de l'animation et du travail social, J. Ion élargit la fonction de ces profes-

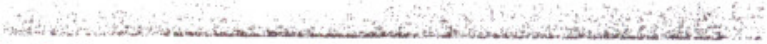

1. Geddes P., Cities in evolution, William Norgate, London, 1915. Sutcliffe A.. Towards the planned city 1880-1914. Basic Blackwell, Oxford, 1981. Gaudin J.P., L'avenir en plan, Mardaga, 1986.

2. Eversley D., The planners in society, the changing roles of a profession, Faber and Faber, London, 1972. Pahl R.H., "Managers, technical experts and the state n, in M. Harloe, Captives Cities, J. Wiley, 1977, p. 491.

3. Bobroff D. Verwaeke, et alii (1981), Réhabilitation des quartiers anciens, Plan construction, 1981.

4. Ion J., "Les animateurs socioculturels en France, limite d'une problématique de la professionnalisation "Loisirs et Société, vol. 5, 1982. Sociabilité et lien social : les intellectuels médiateurs entre l'Etat et la société civile, in Les couches moyennes salariées: mosaïque sociologique. Mission de la recherche urbaine, 1983.

5. Bauer A., Cohen D., Les limites du pouvoir des cadres: l'organisation de la négociation comme moyen d'exercice de la domination. Sociologie du travail 3/1980.

6. Imbert M., Recensement exploratoire sur les professions de l'urbanisme, S F U-D U P, 1979.

Les Annales de la recherche urbaine, no 37

0180-930-X/88/37/98/8 - (C) MELATT/Gauthier-Villars 
sions intermédiaires non seulement à la transmission d'un savoir du bas vers le haut, mais aussi à l'élaboration de savoirs partiels dont la détention est partie prenante de leur rôle de médiation : la mise en relation, l'habileté à constituer les systèmes d'action, la capacité à faire exister une catégorie sociale ou démographique, le discours sur l'harmonie du corps social constituent un moyen d'intervention problématiséd et s.

Les urbanistes, placés en position intermédiaire, notamment entre l'Etat et les collectivités locales, participent à cet interface de l'Etat et de la société civile où se jouent les légitimités de l'intervention urbanistique publique, où se définissent des formulations renouvelées de l'intérêt public (le logement social, l'équipement public, l'usager et la qualité urbaine, le développement social).

De la société civile à l'Etat, les urbanistes participent à l'élaboration et à la formalisation de projets urbanistiques; de l'Etat à la société civile, les urbanistes définissent des modalités d'intervention (plan, programme, stratégies) où les projets participatifs soustendent des projets sociaux. Ainsi peut être envisagée la double face des professionnels de l'urbanisme: à une éthique professionnelle de l'élaboration du projet spatial, de l'étude préalable, se superposent les redéfinitions de la gestion publique, de la gestion sociale. Les urbanistes français remplissent deux catégories de rôles. Aux rôles d'experts centrés sur l'analyse urbaine et la formulation de projets spatiaux s'ajoutent des rôles de médiateurs portés sur l'élaboration de projets sociaux, sur la définition de stratégies de développement urbain et sur les formulations de l'intérêt public qui mobilisent, au-delà des savoir-faire, des compétences de recherche, capacité d'innovation, et aussi une certaine imagination.

Ces faits ont été établis à partir d'une enquête auprès de 200 urbanistes en exercice.

\section{LA PERCEPTION DES RÔLES PROFESSIONNELS}

L'interrogation par questionnaire permet, dans une certaine mesure, de préciser la manière dont les urbanistes perçoivent leurs orientations professionnelles c'est-à-dire leurs préférences à l'intérieur des logiques de l'intervention urbanistique, leurs choix et leurs hiérarchies sur les différentes représentations. La notion d'orientation professionnelle désigne à la fois leur conception privilégiée de l'urbanisme, mais aussi leurs priorités dans l'exercice professionnel, où nous distinguons trois dimensions économiques, spatiales et décisionnelles formulées selon les termes suivants :

\section{$V 15)$ Pour vous quelles sont les exigences prioritaires} de l'urbanisme?

1) Formuler des projets spatiaux en réponse aux exigences sociales ......................... $52 \%$ 2) Relier la ville avec le mouvement économique .. $21 \%$ 3) Assurer un conseil auprès des instances de décision $14 \%$

Dans la structure des perceptions professionnelles, l'orientation spatiale de mise en forme et du projet urbain l'emporte sur l'orientation économique du développement urbain et sur celle de l'aide à la décision. Les modes d'action représentent la part maîtrisée de l'intervention professionnelle. Les répondants formulent une hiérarchie d'actions où dominent les activités de conseil et d'étude, au détriment du contrôle de légalité (question V 11).

V 11) Quel est votre mode d'action à titre principal ? 1) Assistance, conscil ....................... $82 \%$

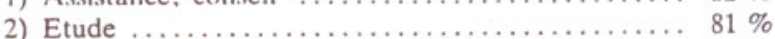

3) Montage opérationnel ........................ $\%$

4) Enseignement, recherche $\ldots \ldots \ldots \ldots \ldots \ldots \ldots, 32 \%$

5) Contrôle de légalité ................... $29 \%$

\section{LA MÉTHODE DE L'ÉCHANTILLONNAGE}

- La méthode d’échantillonnage procède d'un sondage en grappe, selon un choix de région (selon leurs niveaux de développement), puis d'un choix d'agglomération (16 agglomérations). L'échantillon des agglomérations offre une diversité de situations urbanistiques entre les aggiomérations industriclles (Metz-Le Havre-Douai), les villes moyennes (Chambéry, Châlon-sur-Saône, Thiers), les zones à dominante rurale (Foix, Laon).

- Sur chaque agglomération, douze urbanistes ont été interrogés, en respectant la diversité des filières d'exercice. L'enquête porte sur la population des praticiens, cadres de l'urbanisme (évaluée à 3000 en 1979). diplômés ou pas, mais exerçant des missions de l'urbanisme (étude - conseil opérationnel - contrôle) au moins $50 \%$ de leur temps.

- Les filières d'exercice ont été soumises au respect de quotas. afin d'assurer le maximum de fidélité de l'échantillon. Sur la base des effectifs urbanistes établis par le recensement exploratoire S F U/D U P de $1979^{6}$, corrigés par une estimation des recrutements sur chaque filière d'exercice de 1979 à 1985 , nous avons retenu les quotas suivants des filières dexercice Services de l'Etat $(30 \%)$ - Services communaux $(20 \%)$ Matitrise d'ouvrage sociale (H L M - S E M - PACT ARIM)
(9\%) - Urbanistes libćraux $(17 \%)$ - Bureaux et associations d’étude $(13 \%)$ - Organismes sous tutelle pluri-communale $(11 \%)$ : Agence départementale - Agence d'agglomération - Sivom - Conseil Régional.

L'ohjectif de l'enquête est d'examiner les rôles, les attitudes ct les représentiations des professionnels de l'urbanisme it partir de trois variables explicatives: les filières d'exercice, les formations de base, les formations en urbanisme, en gardant le maximum de fidélité au regard d'un recensement national. Ici le taux de sondage (192/4000, environ 1/20) assure une certaine fidélité pour une recherche exploratoire.

Au regard de la précédente étude de $\mathrm{M}$. Imbert ${ }^{6}$ notre échantillon comporte la même structure de formation de base : $41 \%$ architectes - $34 \%$ universitaires $-20 \%$ ingénieurs $-5 \%$ divers. L'élargissement du champ professionnel aux filières communale et pluri-communale a pour effet de valoriser le nombre d'ingénieurs. Le nombre de diplômés en urbanisme (institut, DE S S, maitrise d'aménagement) s'accroît de 50 à $60 \%$. Cette enquête a été financée par le S T U/D T A et la D A U du Ministère de l'Equipement. Y ont participé $\mathrm{M}^{\text {mes }}$ Maurel Journel et A. Lyotard, MM. J.P. Villetird et J.M. Vanhoutte. 
Néanmoins ces rôles médiateurs peuvent être sériés et analysés sur plusieurs dimensions :

$V$ 34) La médiation entre les exigences de l'Etat et les exigences des municipalités (ainsi pensent $65 \%$ des professionnels).

$V$ 35) L'importance des exigences avancées par les habitants et les associations urbaines (ainsi pensent $61 \%$ des professionnels).

$V$ 36) La médiation entre les exigences des maitres d'ouvrage et les exigences des habitants (ainsi pensent $50 \%$ des professionnels).

Ces différentes médiations permettent de reconnaitre les différents types d'exercice de la médiation urbanistique. La délégation d'Etat, accompagnée du contrôle de légalité, a longtemps occulté la possibilité d'un urbanisme de conseil, de revendication et de participation. La promotion de la qualité urbaine auprès des maitres d'ouvrages et des constructeurs implique l'intervention des professionnels entre les exigences des

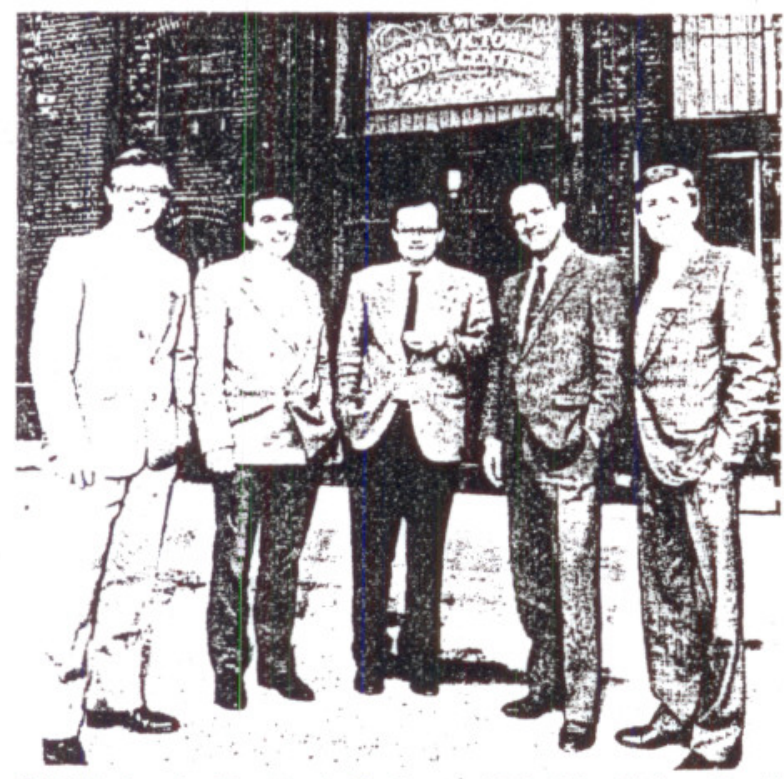

I N T A London Docklands Conference 1987. John Walker, David Locke, M. Dresch, Jacques Guyard, Michael Greenlees.

maitres d'ouvrage et les exigences des habitants. La pression associative tend aussi à redistribuer l'action des professionnels.

Comment se composent ces rôles médiateurs sclon les types d'exercice et selon les modèles de qualification? Ce développement des rôles médiateurs provient-il seulement des mutations dans les politiques urbaines? Quelle part peut-on attribuer au développement de la formation et à la spécialisation croissante de l'enseignement?

\section{MODÈLES DE QUALIFICATION ET MODELES DE MÉDIATION}

La diversité des professions de l'urbanisme et de leurs exercices professionnels peut être décrite non seulement par une liste des positions sociales (statuts, filières d'exercice). Elle doit inclure les modes de référence ${ }^{9}$ et de représentations énoncés et reconnus par les professionnels : modèles d'identification, profils de qualification, modèles de médiation.

Les clivages de statuts (libéral/fonctionnaire) façonnent fortement le champ des interventions urbanistiques. L'orientation spatiale est associée à une identification professionnelle par le diplôme. Il en est ainsi des urbanistes à statut libéral. Les salariés et contractuels de l'urbanisme soutiennent davantage une identification professionnelle par l'expérience sur l'ensemble de l'échantillon. Les praticiens définissent la profession d'urbaniste d'abord par des critères d'expérience $(78 \%)$, puis des critères d'appartenance à une organisation $(40 \%)$ et peu par le critère du diplôme $(20 \%)$.

Ces relations entre les préférences sur le champ d'intervention, les tâches et les profils peuvent être décrits au moyen de la notion de modèle de qualification. La

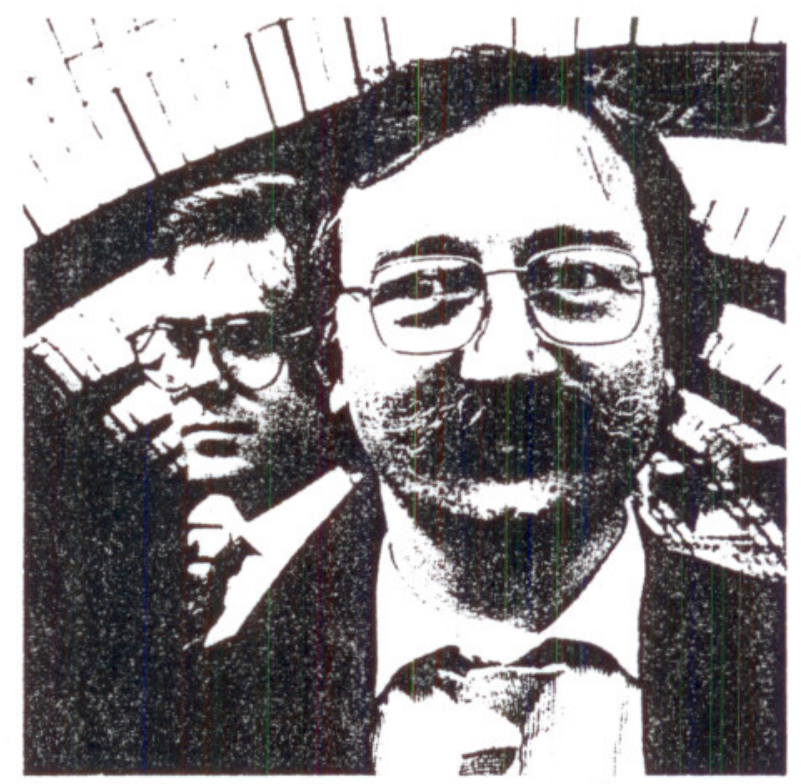

Jacques Lichnerowicz et Jean-Jacques Bravo, organisateurs du Salon international de l'architecture.

sociologie des occupations professionnelles nous apprend que le modèle de qualification dénomme la manière dont la situation de travail est décrite par l'employé ct reconnue par l'employeur. La définition de la situation de travail est le résultat d'un consensus entre les exigences de l'employeur et les exigences de l'employé à partir des jugements de qualification ${ }^{10}$.

Dans cet examen des professions de l'urbanisme, nous avons distingué les modèles de qualification suivants : " chargé d'étude" - "chargé d'opération" " chargé de mission " - " conseil sous statut libéral " — " directeur d'organisme"

9. Merton K.. Eléments de théorie et méthode. Plon. 1969. Bourdieu P. Questions de sociologie. Ed. de Minuit. 1980.

10. Tripier P.. Saussois J.M.. Rivard P.. L'espace de qualification des cadres, Cedress, Paris, 1980. 
La spécification des interventions urbanistiques mises en cuvre contirme cette priorité des activités de conseil, d'étude préalable et d'urbanisme opérationnel, à l'intérieur d'un champ d'action très diversifié.

\section{10) Quel type d'intervention urbanistique mettez-vous en auvre actuellement?}

1) Le conseil aux communes ................. $85 \%$

2) Etude préalable de quartiers nouveaux ( $\mathrm{H} \vee \mathrm{S} /$ F A U/C I V) ......................... $70.6 \%$

3) Urbanisme operationnel ( $\mathrm{Z}$ A C, tinancement, amenagement) .............................. $70.3 \%$

4) Etude préalable de quartiers anciens .........66.8 \%

5) Programmation à moyen terme ( $\mathrm{P} \mathrm{O} \mathrm{S/S} \mathrm{D} \mathrm{A} \mathrm{U)} 63 \%$

6) Intervention sconomique (aide ì l'implantation.

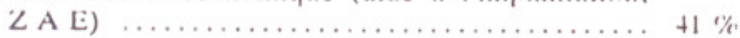

\section{GESTION \\ ET ANTICIPATION}

L'exigence d'anticipation est reconnue par les professionnels au travers d'une diversité des formules opératoires (conseil — programme - stratégie - prévision spatiale). Le recul de la prévision à long terme au profit du conseil et de la stratégie souligne le glissement d'un urbanisme abstrait, vers un urbanisme centré sur les acteurs et leurs stratégies.

$V+3)$ Votre travail conserve-t-il une capaciti d'anticipation? De quel ordre?

1) Conseil à des instances de décision .......... $88 \%$

2) Programmation à moyen terme ............. $77 \%$

3) Définition de choix stratégiques .............. $75 \%$

4) Formulation de projets spatiaux ........... $68 \%$

5) Analyse prévisionnelle ................... $64 \%$

Pour les professionnels du projet, dans leur diversité d'expression (conseil, programme, stratégie, projets spatiaux), il convient de préciser comment les projets composent avec les données du pouvoir et de la décision. La hiérarchie des objectifs de gestion reconnue permet de préciser les exigences de la commande.

V 39) Pouvez-vous préciser les objectifs de gestion dont vous êtes responsables?

1) Gérer l'espace ................ oui $83 \%$ non $15 \%$

2) La gestion d'organisme (administra-

tion) $\ldots \ldots \ldots \ldots \ldots \ldots \ldots \ldots \ldots \ldots$ oui $71 \%$ non $25 \%$

3) La gestion d'opérations de construc-

tion ..................... oui $68 \%$ non $28 \%$

4) L'administration municipale (straté-

gie urbaine) $\ldots \ldots \ldots \ldots \ldots \ldots$ oui $57 \%$ non $37 \%$

5) La gestion du logement (location et

service) $\ldots \ldots \ldots \ldots \ldots \ldots \ldots$ oui $18 \%$ non $72 \%$

La permanence et la priorité de l'orientation spatiale dans les métiers de l'urbanisme est soulignée par une attitude de priorité sur les autres orientations économiques et décisionnelles, par un choix accordant une priorité à l'objectif de gestion de l'espace, par une mise en avant des formules d'anticipation par la définition de stratégies et la formulation de conseil.

Cette orientation spatiale doit coexister avec des orientations plus économiques. Les poids de la gestion d'organisme et de la gestion communale soutiennent des formes d’anticipation liées à la programmation économique.

\section{LES RÔLES MÉDHATEURS DES URBANISTES}

Dans ce travail de concertation, de mise en relation avec les élus, les partenaires de l'opération, il convient de préciser ce qui est en jeu, non seulement l'élaboration d'un projet d'urbanisme, mais au préalable une certaine définition de l'intérêt public. L'Etat et ses services ont, dans le passé, organisé les rôles professionnels autour des procédures de la puissance publique et de leurs délégations. Dans le nouveau contexte, nous devons préciser la place des élus locaux (municipalités, départements, régions) dans ces médiations urbaines ${ }^{7}$, les places respectives des différents partenaires dans la formulation d'un intérêt public. Décrire des modes de formation et de formalisation de l'intérêt général, par les professionnels de l'urbanisme, ne préjuge pas du contenu social de cet intérèt général, différencié selon les situations urbaines et politiques ${ }^{x}$.

Les premiers résultats suggèrent une conscience réelle des professionnels dans leur participation à la formation de l'intérêt public.

V 30) Dans vorre exercice professionnel, vous estimezvous porteur de l'intérêt public?

oui $86 \%$ non $8 \%$ non réponse $4,5 \%$

L'énoncé d'une série de questions précise ce réseau des relations de formation de l'intérêt public dans lequel s'inscrit l'action du professionnel.

V 31) D'après vous, qui formule l'intérêt public à titre principal?

1) Les élus locaux ......64\% oui $15 \%$ non $20 \%$ n.r.

2) L'administration d'Etat $48 \%$ oui $27 \%$ non $25 \%$ n.r.

3) Le gouvernement .......32\% oui $37 \%$ non $31 \%$ n.r.

4) Les commanditaires de

l'étude ............18\% oui $46 \%$ non $36 \%$ n.r.

Les praticiens perçoivent ces nouvelles responsabilités dévolues aux municipalités par les réformes de la décentralisation. Les nouvelles procédures et les nouvelles modalités d'intervention, privilégiant la coordination des acteurs de l'urbanisme et leur contractualisation, engagent l'action et le discours pour les professionnels sur un réseau élargi de relations de concertation.

Tels les chefs de projets dans la mise en œuvre du développement social des quartiers. Tels les animateurs des chartes dans les actions intercommunales. Telles les actions urbanistiques à financements multiples engageant plusieurs structures autonomes.

7. Verpraet G., Mission - Profils et formation de l'urbaniste, S T U. D A U, 1985.

8. Knox P., Planners as urban managers : an exploration of the attitudes and image of senior british planners, Environment and Planning, 13, 1981, p. $881-891$. 
LES FORMULATIONS DE L'INTÉRÊT PUBLIC SELON LES PROFILS DE OUALIFICATION

\begin{tabular}{|c|c|c|c|c|c|c|}
\hline . & 通怤 & 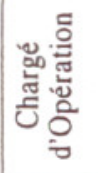 & 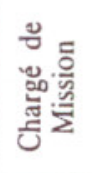 & 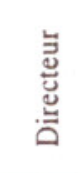 & 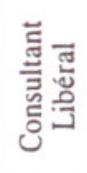 & 홍 \\
\hline $\begin{array}{l}\text { - Vous estimez-vous porteur de l'intérêt public? } \\
\text { v128/ }\end{array}$ & $85 \%$ & $93 \%$ & $97 \%$ & $90 \%$ & $83 \%$ & $87 \%$ \\
\hline $\begin{array}{l}\text { - Selon vous, qui formule l'intérêt public? } \\
\text { v129-l'Etat } \\
\text { v130-le gouvernement } \\
\text { v131-la commande d'étude? } \\
\text { v132-les élus locaux? }\end{array}$ & $\begin{array}{l}43 \% \\
36 \% \\
22 \% \\
58 \%\end{array}$ & $\begin{array}{l}38 \% \\
24 \% \\
27 \% \\
79 \%\end{array}$ & $\begin{array}{l}65 \% \\
24 \% \\
19 \% \\
81 \%\end{array}$ & $\begin{array}{l}56 \% \\
31 \% \\
16 \% \\
68 \%\end{array}$ & $\begin{array}{l}35 \% \\
17 \% \\
17 \% \\
57 \%\end{array}$ & $\begin{array}{l}48 \% \\
32 \% \\
18 \% \\
64 \%\end{array}$ \\
\hline $\begin{array}{l}\text { - Pensez-vous que les élus soient capables de formuler l'intérêt public ? } \\
\text { v133/ nou }\end{array}$ & $\begin{array}{l}67 \% \\
15 \%\end{array}$ & $\begin{array}{l}68 \% \\
14 \%\end{array}$ & $\begin{array}{r}82 \% \\
3 \%\end{array}$ & $\begin{array}{l}70 \% \\
11 \%\end{array}$ & $\begin{array}{l}70 \% \\
10 \%\end{array}$ & $\begin{array}{l}68 \% \\
14 \%\end{array}$ \\
\hline $\begin{array}{l}\text { - Pensez-vous que l'administration formule mieux l'intérêt public que } \\
\text { les élus locaux? } \\
\begin{array}{ll}\text { v134/ } & \text { - en général } \\
\end{array}\end{array}$ & $\begin{array}{l}16 \% \\
43 \%\end{array}$ & $\begin{array}{l}14 \% \\
28 \%\end{array}$ & $\begin{array}{r}9 \% \\
46 \%\end{array}$ & $\begin{array}{l}17 \% \\
51 \%\end{array}$ & $\begin{array}{l}17 \% \\
39 \%\end{array}$ & $\begin{array}{l}29 \% \\
42 \%\end{array}$ \\
\hline $\begin{array}{l}\text { - Vous estimez-vous médiateur entre les exigences de l'Etat et celles } \\
\text { des collectivités locales? } \\
\text { v135/ }\end{array}$ & $65 \%$ & $34 \%$ & $69 \%$ & $66 \%$ & $64 \%$ & $65 \%$ \\
\hline $\begin{array}{l}\text { - Les exigences des habitants et des associations sont-elles importantes } \\
\text { dans votre pratique professionnelle? } \\
\text { v136/ }\end{array}$ & $61 \%$ & $\begin{array}{l}73 \% \\
27 \%\end{array}$ & $\begin{array}{l}80 \% \\
16 \%\end{array}$ & $\begin{array}{l}59 \% \\
34 \%\end{array}$ & $\begin{array}{l}56 \% \\
33 \%\end{array}$ & $.61 \%$ \\
\hline $\begin{array}{l}\text { - Vous estimez-vous médiateur entre les exigences des habitants et } \\
\text { celles des maitres d'ouvrage? } \\
\mathrm{v} 137 /\end{array}$ & $47 \%$ & $49 \%$ & $57 \%$ & $35 \%$ & $66 \%$ & $50 \%$ \\
\hline
\end{tabular}

d'action est composé par les différentes institutions de l'urbanisme, mais aussi par les rapports de dépendance et de concertation, noués entre les acteurs de l'urbanisme dans un contexte d'intervention. Dans ce cadre d'analyse et à l'intérieur de la perception des acteurs, des zones de compétence peuvent être définies comme autant d'expertises justifiées ${ }^{12}$. Cette dialectique étroite entre les rôles d'experts et les rôles de médiateurs conduit à des compromis multiples, relativement stables, qui sont figurés et décrits dans les modèles de qualification: chargé de mission, chargé d'étude, chargé d'opération, architecte consultant...

Ces modèles de qualification sont autant de manière d'agencer les différentes relations de médiation et les types d'expertise requis par l'intervention urbanistique (cf. tableau 2).

Le concept de médiation peut être décrit comme un système de négociation noué à l'intérieur d'un processus où se développent des argumentations, des stratégies et des transactions ${ }^{13.14}$. Dans le cas des urbanistes français, le concept de médiation est construit comme un système de positions intermédiaires, où dominent les relations entre l'Etat central et les collectivités locales, entre les maîtres d'ouvrage et les élus locaux. Les négociations sur les orientations, les transactions sur le projet urbain, les choix des moyens se développent à l'intéricur de ce système d'action.

\section{LES MODÉLES DE MÉDIATION}

Cette analyse des relations entre les orientations professionnelles, les rôles médiateurs et les modèles de qualification permet de mieux saisir les différents moments de la médiation dans l'urbanisme français, comme autant de déconstruction d'une idée homogène de l'intervention technique.

L'Etat central s'est constitué en médiateur urbain, dans les années 1940-50 sur les pratiques de reconstruction des villes détruites et face à l'urbanisation massive des années 50. Cette gestion centrale de l'urbanisme a été organisée par les professions de fonctionnaires (les

12. Benveniste G., The politics of expertise, Glendary Press, Berkeley, 1972.

13. Forrester J., What are the planners up against? Planning in the face of power. The Bulletin of the Associate School of Planners, vol. XVIII, $1980, \mathrm{n}^{\circ} 2$

14. Susskind $\mathrm{L}$. and Ozawa $C$. Mediated negotiation in the public sector : the planners as mediator, Bulletin of A CS P, 1984, no 3. 
102 plans et projers

Ces profils de qualification ne désignent pas simplement des statuts professionnels plus ou moins reconnus. Ils décrivent aussi des modes d'agencement distincts des rôles professionnels. Les profils rattachés à l'expertise spatiale mettent en avant les tâches du projet spatial. Les profils rattachés à l'expertise de programme privilégient les tâches du programme (définition du choix stratégique, montage financier) devant l'élaboration du projet.

\section{LES MODĖLES DE QUALIFICATION}

Dans cet examen de contenu des profils de qualification, nous nous attacherons à décrire l'agencement des rôles gestionnaires et des rôles médiateurs.

\section{- Les profils liés à l'intervention sur l'espace :}

- Le modèle du conseil libéral (23\% de l'échantillon) est lié au statut d'exercice indépendant et à la formation de base en architecture. Il accorde une importance centrale aux préoccupations spatiales $(58 \%)$ mais néglige l'aide à la décision $(8 \%)$. Il privilégie parmi les rôles médiateurs la relation entre les maîtres d'ouvrage et les habitants (V $137: 66 \%$ oui). Distants des tâches de gestion et des systèmes de décision, ils accordent la priorité à l'anticipation par les moyens du projet spatial. Cette revendication privilégiée de l'expertise spatiale participe au modèle traditionnel de l'architecte urbaniste.

- Le modèle du "chargé d'opération" (9\% de l'échantillon) diffuse dans les organismes chargés de la gestion de l'habitat, mais aussi dans les municipalités et les bureaux d'étude, sur des tâches de montage d'opération et de promotion des qualités urbaines. Il accorde la priorité aux interventions spatiales $(47 \%)$ et aux préoccupations économiques (35\%). Ce profil de qualification est lié à l'urbanisme opérationnel, surtout aux interventions en quartiers anciens, notamment sur des tâches de montage d'opération. Cette position de gestion opérationnelle soutient une relation plus directe aux exigences des habitants (V 136 : $73 \%$ ) par le truchement des études de la demande et des enquêtes de besoins.

\section{- Profils privilégiant urbanisation et mutations économiques}

- Le modèle de "chargé d'étude " (49\% de l'échantillon) s'est constitué autour des tâches spécifiques de l'analyse urbaine, des études préalables aux implantations, des analyses de prévision et de programmation, particulièrement dans les services de l'Etat et ses délégations départementales, agences d'urbanisme et bureaux d'étude. Ce profil privilégie les orientations spatiales $(53 \%)$ et les orientations économiques (20\%).

Ces rôles médiateurs sont concentrés sur la relation entre les exigences de l'Etat central et les exigences des collectivités locales (V 135: $65 \%$ ).

L'importance accordée aux élus locaux dans la formation de l'intérêt public (V 132 : $58 \%$ ) est contre- balancée par un recours affirmé à l'Etat dans certains cas (V 134: $43 \%$ ). Ces chargés d'étude semblent relativement distants des exigences des maitres d'ouvrage (V $137: 47 \%$ ).

\section{- Les profils privilégiant l'aide}

à la décision

- Le profil de chargé de mission (11\%) est un nouveau profil qui se développe au sein des administrations de conseil, sous la responsabilité de plusieurs municipalités (administrations régionales, agences départementales d'urbanisme, syndicats intercommunaux). Avec une préférence pour l'aide à la décision (30\% contre $15 \%$ pour l'ensemble de l'échantillon), ils remplissent leurs rôles médiateurs d'abord avec les élus locaux (V 133 : $82 \%$ ), puis avec les habitants (V 132: $80 \%$ ).

Leur capacité d'anticipation est plus centrée sur une élaboration stratégique que sur une formulation du projet spatial. Leurs tâches consistent d'abord à définir les objectifs sociaux et politiques pour les élus, à mobiliser les ressources (financement, équipe de consultants, montage d'opération) à l'intérieur de stratégies à moyen terme. De cette manière, la gestion stratégique pénètre à l'intérieur des tâches de conception et préfigure de nouvelles pratiques urbanistiques.

- Les deux profils de chargé de mission et de directeur sont proches dans l'univers des pratiques. Mais seul le profil de directeur maintient un équilibre entre les trois champs de préoccupations (économiques, spatiales et décisionnelles), entre les rôles gestionnaires et les rôles médiateurs, entre les exigences de l'Etat central et les exigences des collectivités locales.

L'analyse des modèles de qualification permet de préciser le déplacement des références professionnelles, des modèles de professionnalisation et des modèles de médiation. Le modèle de l'expert spatial est caractérisé par une efficacité attendue de savoirs urbanistiques spécitiques, et par une identification professionnelle fondée sur le diplôme d'urbaniste ${ }^{11}$.

A ce type de performance et d'expertise professionnelle s'ajoutent maintenant des modèles de médiation plus centrés sur les relations de concertation, de prise en compte des acteurs de l'urbanisme et de soutien à l'aide à la décision. Les modèles de chargé de mission et de chargé d'opération décrivent ces nouveaux contenus. Dans cette interprétation, l'intervention sur l'espace reste une priorité $(50 \%)$, mais les stratégies de professionnalisation composent avec les modèles de médiation plus affirmés.

\section{LES RELATIONS DE L'EXPERT ET DU MÉDIATEUR}

Dans notre approche, la notion de médiation désigne de manière descriptive et normative le système d'action, où peut se développer l'action des professionnels et l'intervention de leurs techniques propres. Ce système

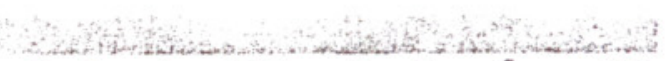

11. Parsons T., Essays in social theory, Free Press, Glencoe, 1958. 
104 plans et projets

urbanistes de l'Etat, les ingénieurs des Ponts) et leurs délégations (notamment l'ordre des architectes) dans les années 1943-1968. Dans ce contexte des politiques urbaines de l'Etat, l'élaboration du projet spatial est insérée dans une programmation à moyen terme (10 ans pour les communes, 25 ans pour les agglomérations). Pour assurer cet exercice professionnel, s'est constitué un modèle spécifique de qualification, dénommé " chargé d'étude ", qui représente actuellement $49 \%$ de la profession. Sa caractéristiqué est celle d'un agencement étroit des expertises spatiales et des expertises économiques.

Les années de crise économique, les pratiques de réhabilitation des quartiers anciens ont renforcé la médiation et l'intervention des promoteurs sur l'urbanisme. Les exigences nouvelles des habitants sont prises en compte dans le cadre d'une gestion stratégique où se superposent les objectifs d'économie d'énergie, de qualité intérieure du logement, de définitions de nouveaux espaces publics aux abords du bâtiment. Là réside le travail spécifique des chargés d'opération dans le montage des programmes de logement. Dans cet assouplissement de l'intervention normative de l'Etat, les architectes urbanistes ont essayé de redéfinir l'insertion urbaine des bâtiments dans une relation plus étroite entre la typologie du logement et la morphologie urbaine. Cette tendance porte une séparation des expertises économiques et des expertises spatiales.

Les réformes de la décentralisation (1983-85) tentent de promouvoir la médiation municipale par un déplacement des médiations de l'Etat. Les interventions municipales et régionales traitent de la participation du public, de la cohabitation entre résidents, de la symbolique des espaces publics. Pour les chargés de mission et les chargés d'opération, l'élaboration du projet d'urbanisme reste dans une formulation stratégique. L'exigence municipale ajoute une volonté de coordonner les acteurs de l'urbanisme au niveau municipal (recherche des synergies), de prendre mieux en compte les exigences de la gestion sociale dans les quartiers de logements publics. Cette convergence locale de différents organismes permet un financement d'actions urbanistiques par plusieurs structures, mais elle accroît les exigences de médiation et de coordination entre les différents acteurs de la ville. Telles les procédures du développement social des quartiers, tels les programmes de développement local de la région Midi-Pyrénées, telles les diverses actions intercommunales où les profils de médiateurs complètent ces multiplicités d'expertises économiques, spatiales, sociales, organisationnelles, politiques.

\section{L'ENSEIGNEMENT DE L'URBANISME}

Pour évaluer le rôle de la formation dans la composition d'une culture professionnelle, il nous paraît intéressant de distinguer la part de la formation de base plus centrée sur l'acquisition de connaissances à l'intérieur de disciplines (économie, architecture, sociologie) diverses et la part de la discipline urbanistique, plus orientée vers une aptitude à la synthèse. Dans ce deuxième temps de la formation peuvent s'apprendre les démarches de l'urbanisme compréhensif, celles de l'élaboration du projet spatial, celles de l'apprentissage des rôles médiateurs.

L'enquête confirme comment la formation urbanistique prépare d'abord aux compétences de l'expertise spatiale. Mais les rôles médiateurs peuvent être acquis autant par une formation urbanistique, que par une expérience professionnelle motivée à ces tâches.

\section{FORMATION DE BASE ET EXPERTISE}

Le champ professionnel de l'urbanisme recrute dans différentes formations de base (architecte, ingénieur, maîtrise en sciences sociales) où se forme et se reproduit une hiérarchie sociale. Les urbanistes issus d'une formation architecturale occupent principalement le profil de consultant libéral $(80 \%)$ et une part du profil de chargé d'étude (37\%). Les urbanistes issus d'une formation en sciences sociales $(30 \%)$ se destinent au profil de chargé d'étude $(46 \%)$ et maintenant aux profils de chargé de mission (23\%) ; de chargé d'opération $(23 \%)$.

A côté d'une conception architecturale de l'urbanisme, centrée sur le projet spatial, à côté d'une formation à la planification pour les ingénieurs basée sur les techniques urbaines, l'intervention des sciences sociales au service de l'enquête préalable et de l'analyse urbaine a redéfini sa place dans l'élaboration programmatique des modes de vie. C'est un des résultats de l'extension du profil de chargé d'étude $(49 \%)$.

\section{FORMATION URBANISTIQUE ET QUALIFICATION}

L'objet principal des instituts d'urbanisme en France (deux années d'étude après la maîtrise) est de développer une aptitude à la synthèse (1), à comprendre les faits urbains (géographique, économique, sociologique) et à conduire l'élaboration d'un projet spatial (1). De cette manière ont été préparés les profils de chargé d'étude qui étaient en grande demande pendant les années de croissance urbaine (1960-70).

Les résultats de l'enquête auprès des praticiens montrent comment l'acquisition du diplôme d'urbaniste et son utilisation professionnelle accroissent la perception de l'identité professionnelle. Cette formation se préoccupe d'abord de l'élaboration du projet spatial (48\%) puis du soutien au développement économique (31\%), mais elle ne prépare guère aux tâches préparatoires à la décision $(15 \%)$.

\section{LA PRÉPARATION AUX RÔLES MÉDIATEURS}

Les diplômés en urbanisme semblent relativement motivés pour défendre l'intérêt public $(84 \%)$ et ont conscience de leurs rôles médiateurs entre les exigences de l'Etat et les exigences des municipalités (69\%). Cette motivation à défendre l'intérêt public (87\%) 
experts ou médiateurs? 105

dans la médiation centrale/locale $(71 \%)$ est également importante pour les praticiens non diplômés en urbanisme. L'identification professionnelle par l'expérience accroît la priorité accordée dans les orientations professionnelles à l'aide à la décision. Car l'expérience professionnelle est celle de l'insertion dans des organismes d'urbanisme, spécialement dans des organismes publics (administration municipale, régionale, étatique). L'enseignement de l'urbanisme contribue à l'acquisition de capacités d'expertise pour l'élaboration du projet spatial, pour l'aide au développement économique. La préparation aux rôles médiateurs dépend des expériences professionnelles et de cours complémentaires en sciences politiques.

Il en résulte un processus de formation à l'urbanisme en trois étapes ${ }^{15}$ :

- une formation de base donne l'accès à des qualifications disciplinaires au niveau de la maitrise : le droit et la norme, la sociologie et l'enquête etc.,

— l'éducation d'urbaniste prépare à l'expertise spatiale. - l'acquisition des rôles médiateurs relève des expériences professionnelles ou d'un enseignement complémentaire en sciences politiques ${ }^{16}$.

Reste un problème particulier pour les pédagogues de l'urbanisme. Comment préparer les futurs professionnels à leurs rôles de médiateurs dans la participation du public, face au pouvoir des maires sur la planification urbaine, en relation aux nouvelles administrations régionales? Différentes solutions partielles sont avancées par les pédagogues de l'urbanisme ${ }^{17}$ :

- accroître les cours de sciences politiques et de gestion publique,

- développer les ateliers et les stages sur l'élaboration des stratégies urbaines,

- renforcer les relations entre les instituts d'urbanisme et les élus locaux.

L'évolution constatée des profils professionnels de l'urbanisme provient d'abord des changements des politiques urbaines, du rôle accru des élus locaux, du réagencement des rapports entre les acteurs de l'urbanisme et des nouvelles modalités d'intervention des organismes. Le rôle des instituts d'urbanisme est porté, face à ces changements, à maintenir une tradition, celle de l'urbanisme compréhensif, à soutenir une exigence, celle des études préalables et à reformuler quelque peu ces fondements, au regard des nouveaux modèles de chargé de mission et de chargé d'opération. Dans notre approche des professions de l'urbanisme et de leur qualification, c'est le rôle des formations en urbanisme d'expliciter ces réagencements de pratiques et ces positions intermédiaires afin de contribuer à la formulation des éthiques médiatrices et à leur renouvellement. Les demandes de formation permanente en terme de communication, d'animation ne traduisent qu'une interprétation réduite de ces redéfinitions professionnelles.

Le réagencement des rapports entre experts et médiateurs participe d'une conception élargie de la praxis urbanistique, où l'instrumentalité technique est replacée dans les processus d'interaction sociale et de mobilisation d'acteurs, et laisse ainsi place aux formulations symboliques de ces processus urbains. Nous savons tous que les plans d'urbanisme ne sont pas de simples instruments de production de l'espace, de redisposition des réseaux techniques, mais impliquent les groupes sociaux résidents et les significations qu'ils attachent à l'espace.

Ces éthiques médiatrices peuvent porter sur plusieurs formules entre les médiations politiques (élus, stratégies, Etat), et les médiations sociales et culturelles (le projet architectural - les communications urbaines). Mais leur explicitation reste une condition nécessaire de la communication du projet urbain.

\section{Gilles Verpraet}

15. Falludi A.S.. Essays on planning theory and educution, Pergamon, London, 1978

16. Witch F. The political world of urban planners, Bulletin of A C S P , 1984, n³

17. Habermas J., Connaissance et intérèt, Payot, 1973 ; Théorie de l'agir communicationnel, Fayard, 1987.

Gilles Verpraet, sociologue et urbaniste, est chargé de recherches au CN R S. Ses travaux sur les processus de décision (La Défense, les villes nouvelles, le Canal Rhin-Rhône, l'ont conduit à étudier plus précisément les formations et les qualifications professionnelles des urbanistes. Il a publié sur ce sujet trois rapports de recherches : Les transactions économiques, juridiques et morphologiques dans l'innovation architecturale, C E D R E S S, 1982, Missions, profils et formation de l'urbanisme, S T U, 1986 et L'espace de qualification des urbanistes, D A U, 1987. Il travaille également sur le problème de la cohabitation résidentielle. Il participe aux travaux du CEDRES S. Centre de recherche de I'Université Paris X-Nanterre, et de I'I P R A US, laboratoire de recherche participant à la commission transversale Architecture, urbanistique et société du CNRS. 


\section{(ESA) RECHERCHE URBAINE}

ONT PARTICIPÉ À CE NUMÉRO :

Henri Raymond, Université Paris X ; Anne Vernes-Moudon, Université de Washington à Seattle : Cynthia Ghorra-Gobin, urbaniste ; Olivier Soubeyran, Université de Montréal ; Hans Böhm, Université de Bonn ; Viviane Claude, Délégation à la Recherche et à I'Innovation; Rémi Baudoui, I U P . Université Paris XII ; Albert Lévy, L A T M U T - Université Paris VIII ; Julia Trilling, Massachusetts Institute of Technology; Tadeusz Markowski, Université de Lodz; JeanClaude David, Maison de l'Orient méditerranéen-Université Lyon II ; Jean Gohier, urbaniste ; Gilles Verpraet, I P R A U S ; Pierre Lassave, C E T U R ; Jean-Pierre Gaudin, Plan urbain - L A T M U T ; Jean-Loup Gourdon, Plan urbain ; Anne Charreyron-Perchet, Plan urbain ; Claire Gillio, Plan urbain.

Publié par : Secrétariat permanent du Plan urbain, 64, rue de la Fédération, 75015 Paris. Tél. : (1) 46.47 .32 .71 et (1) 46.47.32.82.

Rédaction: Anne Querrien ; Fabrication : Florence Pétry ; Iconographie: Martine Vernier; Conception graphique : Franck Novati; Secrétariat: Martine Vernier.

\section{CRÉDITS PHOTOGRAPHIQUES :}

Roger-Viollet, pp. 4, 7 ; Massachussetts Institute of technology, pp. 11, 12, 13, 14, 15, 18; C. Ghorra-Gobin, pp. 21, 23; Gilles Peress (Magnum), p. 32 ; Erich Hartmann (Magnum), p. 35 ; Archives municipales de Strasbourg, p. 42 ; Henri Cartier-Bresson (Magnum), p. 43 ; Fondation Le Corbusier, pp. 47, 49, 51 ; Marie-Claire Bordaz, pp. 71, 73, 75; Mark Marian Schmidt (Rapho), pp. 79, 83 ; Gennaro Ghirardelli, pp. 86, 92 ; Jean-Claude David (CNRS-I R M A C, Lyon), pp. 87, 89 ; I A U R I F, pp. 95-96 ; London Docklands development corporation, p. 101 (gauche) : Martin Fraudreau, p. 101 (droite); Margerin-Fromental, p. 112 ; Banlieues 89, p. 118.

Couverture: Projet de Le Corbusier pour Rio de Janeiro (Fondation Le Corbusier).

\section{COMITÉ DE LECTURE :}

Paul Allies, Université de Montpellier ; André Bruston, Ministère de l'Equipement, du Logement, de l'Aménagement du Territoire et des Transports ; Hervé Dupont, Etablissement public de Cergy-Pontoise ; Gabriel Dupuy, Institut d'urbanisme de Paris-Créteil ; Jean-Pierre Gaudin, Université ParisVII ; André Guillerme, Centre national de la Recherche Scientifique ; Jean-Paul Laborie, Université de Toulouse le Mirail ; Yves Prats, Université d'Aix-en-Provence ; Marcel Roncayolo, Université de Paris X-Nanterre ; Marion Ségaud, Centre national de la Recherche Scientifique ; Alain Thiébaut, Institut français d'architecture ; Pierre Veltz, Ecole nationale des Ponts et Chaussées. 